Article

\title{
Live Cell FRET Imaging Reveals Amyloid $\beta$-Peptide Oligomerization in Hippocampal Neurons
}

\author{
Yang Gao ${ }^{1}\left(\right.$, Stefan Wennmalm ${ }^{2}$, Bengt Winblad ${ }^{1,3}$, Sophia Schedin-Weiss ${ }^{1}$ and Lars O. Tjernberg ${ }^{1, *}$ \\ 1 Department of Neurobiology, Care Sciences and Society, Division of Neurogeriatrics, Karolinska Institutet, \\ 17164 Solna, Sweden; yang.gao@ki.se (Y.G.); bengt.winblad@ki.se (B.W.); \\ sophia.schedin.weiss@ki.se (S.S.-W.) \\ 2 SciLifeLab, Department of Applied Physics, Biophysics, Royal Institute of Technology, 17165 Solna, Sweden; \\ stewen@kth.se \\ 3 Theme Inflammation and Aging, Karolinska University Hospital, 14186 Huddinge, Sweden \\ * Correspondence: lars.tjernberg@ki.se
}

Citation: Gao, Y.; Wennmalm, S.; Winblad, B.; Schedin-Weiss, S.; Tjernberg, L.O. Live Cell FRET Imaging Reveals Amyloid $\beta$-Peptide Oligomerization in Hippocampal Neurons. Int. J. Mol. Sci. 2021, 22 4530. https://doi.org/10.3390/ ijms22094530

Academic Editor: Marco Diociaiuti

Received: 15 March 2021

Accepted: 24 April 2021

Published: 26 April 2021

Publisher's Note: MDPI stays neutral with regard to jurisdictional claims in published maps and institutional affiliations.

Copyright: (c) 2021 by the authors. Licensee MDPI, Basel, Switzerland. This article is an open access article distributed under the terms and conditions of the Creative Commons Attribution (CC BY) license (https:// creativecommons.org/licenses/by/ $4.0 /)$.
Abstract: Amyloid $\beta$-peptide $(A \beta)$ oligomerization is believed to contribute to the neuronal dysfunction in Alzheimer disease (AD). Despite decades of research, many details of $A \beta$ oligomerization in neurons still need to be revealed. Förster resonance energy transfer (FRET) is a simple but effective way to study molecular interactions. Here, we used a confocal microscope with a sensitive Airyscan detector for FRET detection. By live cell FRET imaging, we detected A $\beta 42$ oligomerization in primary neurons. The neurons were incubated with fluorescently labeled $A \beta 42$ in the cell culture medium for $24 \mathrm{~h}$. A $\beta 42$ were internalized and oligomerized in the lysosomes/late endosomes in a concentration-dependent manner. Both the cellular uptake and intracellular oligomerization of $A \beta 42$ were significantly higher than for $\mathrm{A} \beta 40$. These findings provide a better understanding of $\mathrm{A} \beta 42$ oligomerization in neurons.

Keywords: amyloid $\beta$-peptide; oligomerization; aggregation; FRET

\section{Introduction}

Alzheimer disease (AD) is the most common cause of dementia, and affects mainly older people [1]. It is projected to affect more than 100 million people worldwide in 2050, and its pathogenesis remains unclear [2]. The aggregation of the amyloid $\beta$-peptide $(A \beta)$ is believed to play a crucial role in the pathogenesis of $\mathrm{AD}[3]$.

$A \beta$ is a 38-43 amino acid peptide cleaved from a transmembrane protein, the $A \beta$ precursor protein (APP) [4]. It is generated intracellularly and secreted to the extracellular space $[5,6]$. Among the various $A \beta$ isoforms, $A \beta 42$ is more toxic than other species because it is more prone to aggregate [7]. A $\beta$ oligomers-both intracellular and extracellularare suggested to impair synaptic function and contribute to cognitive decline during the early stages of $\mathrm{AD}$ [8]. Increased intracellular $\mathrm{A} \beta 42$ has been shown to correlate with $\mathrm{AD}$ neuropathology $[9,10]$. However, more studies on intracellular $A \beta$ oligomers are needed to understand the pathogenesis in early AD.

Oligomeric $A \beta$ exist in a dynamic equilibrium, which is sensitive and influenced by the environment, and biophysical analysis can affect the oligomers [11,12]. Several techniques have been used for studying $\mathrm{A} \beta$ oligomerization, such as Thioflavin $\mathrm{T}$ assays, SDS polyacrylamide gel electrophoresis (PAGE), and fluorescence microscopy [13]. However, only a few of them are suitable to use in living cells under physiological conditions.

Förster resonance energy transfer (FRET) is widely used to measure protein interactions in molecular biology. Due to its strong dependence on the distance between the donor and acceptor [14], it has been used for studying cellular $A \beta$ oligomerization and showed unique capability [15]. 
Several studies have shown that extracellular $\mathrm{A} \beta$ could be internalized and form aggregates in cells $[15,16]$. However, some key questions regarding the fate of internalized $A \beta$ still need to be addressed, such as under what conditions, in which subcellular compartments, and to what extent $\mathrm{A} \beta$ could oligomerize in neurons.

In this study, we performed live cell FRET imaging to detect $A \beta 42$ oligomerization in primary neurons, by using a high-resolution confocal microscope with a sensitive Airyscan detector. We found that monomeric $\mathrm{A} \beta$ could be taken up into primary neurons and oligomerize mainly in the lysosomes and/or late endosomes in a concentrationdependent manner.

\section{Results}

\subsection{Internalized A $\beta$ Oligomerizes in Lysosomes/Late Endosomes}

Fluorescence correlation spectroscopy (FCS), fluorescence cross-correlation spectroscopy (FCCS), and the combination of FRET and FCS (FRET-FCS) were used to investigate the oligomerization state of $A \beta$ in the cell culture medium. These techniques have been shown capable of detecting $A \beta$ oligomers in solution and FRET-FCS can even detect FRET-active oligomers at fractions below $1 \%$ compared to monomers [17]. We prepared $500 \mathrm{nM}$ HiLyte $^{\mathrm{TM}}$ Fluor 488 (HF488) A $\beta 42$ and 500 nM HiLyte ${ }^{\mathrm{TM}}$ Fluor 647 (HF647) A $\beta 42$ in the cell culture medium. The FCCS analysis showed that $A \beta$ aggregation was low in the medium after $24 \mathrm{~h}$ (Figures 1 and S1-S3).

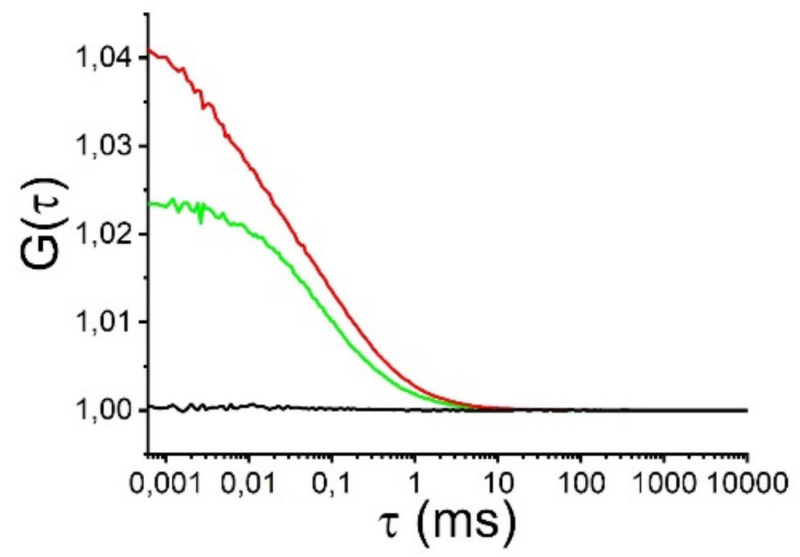

Figure 1. Fluorescence cross-correlation spectroscopy (FCCS) measurements of a sample consisting of 500 nM HF488 A 342 (green) + 500 nM HF647 A 342 (red). Auto- (green and red) and cross-correlation (black) curves were collected in 10-minute measurements after $24 \mathrm{~h}$ incubation. Laser power: $0.4 \%$ $488 \mathrm{~nm}$ and $1.2 \% 633 \mathrm{~nm}$. The lack of cross-correlation indicates that a very low number of oligomers, if any, were present. If there were, the black cross-correlation curves would have an amplitude rising above the 1.0-line, like the green and red auto-correlation curve.

Sensitized emission FRET was used to detect $A \beta$ oligomerization in primary neurons (Figure 2a). In this approach, we excited the donor HF488 A $\beta 42$, which transfers energy directly to the acceptor HF647 A 342 if it is within $10 \mathrm{~nm}$ distance, resulting in emission from the acceptor. The emitted light is then detected by a confocal microscope using an emission filter for the acceptor. Theoretically, it can be excluded that FRET arises due to the low mean monomer-to-monomer distance of $A \beta$ even at high concentration. Thus, the FRET signal in this study indicates $A \beta$ oligomerization. 

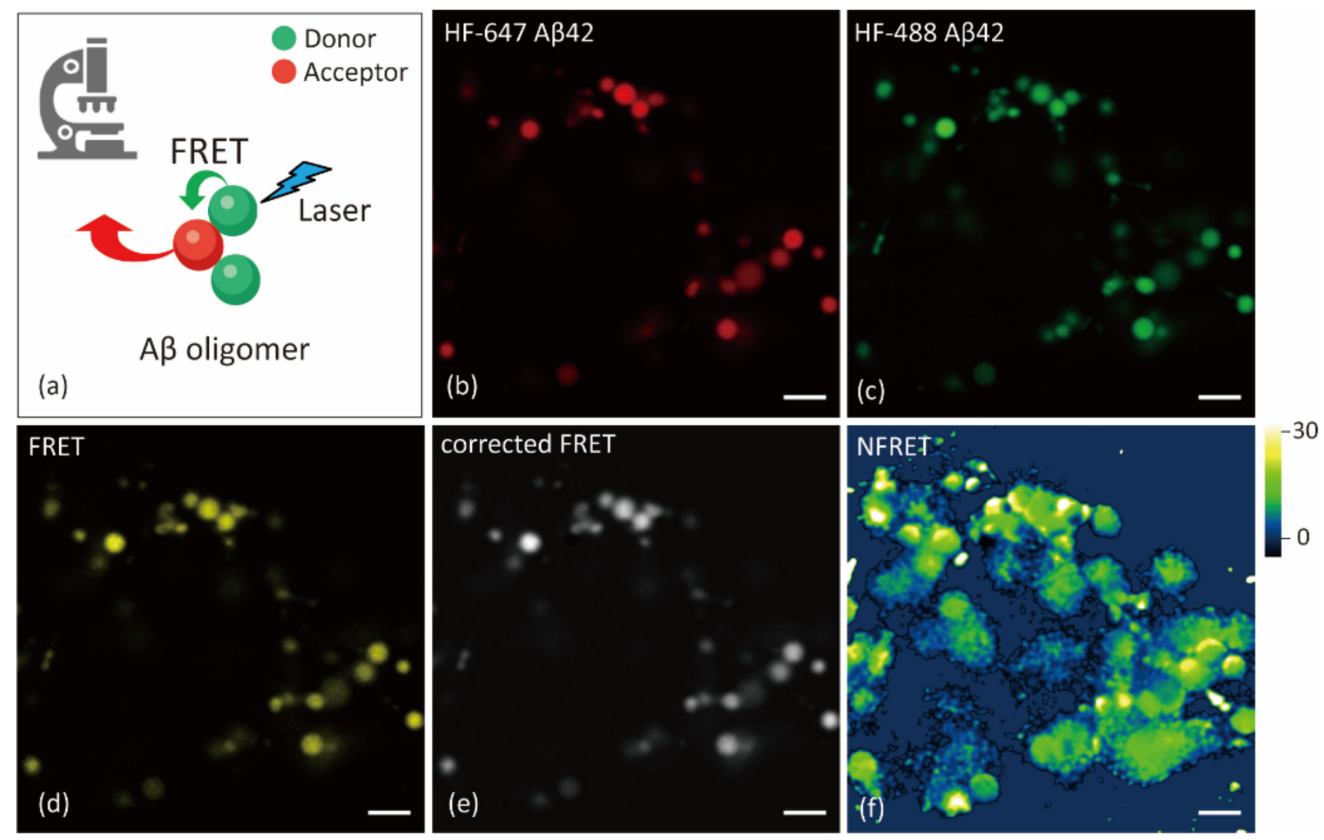

30

Figure 2. Förster resonance energy transfer (FRET) analysis to detect aggregated A $\beta 42$ in primary neurons. (a) Principle of FRET. Equimolar A 342 monomers labeled with HF488 and HF647 are used for oligomerization detection. If the emission of excited donor overlaps with the absorption of the acceptor, energy transfers from donor to ground state acceptor when their distance is $10 \mathrm{~nm}$ or less. (b-d) Acceptor channel (640 nm excitation, 650-700 nm emission), donor channel (488 nm excitation, 480-520 nm emission) and FRET channel (488 nm excitation, 650-700 nm emission) of representative FRET image from confocal microscope. (e-f) The corrected FRET and normalized FRET (NFRET) image from ImageJ PixFRET plug-in. Scale bar: $2 \mu \mathrm{m}$.

It should be noted that fluorescence crosstalk from donor, cross-excited acceptor, and noises such as autofluorescence may interfere with the analysis. To acquire the corrected FRET from crosstalk, spectral bleed-through parameters of the donor and acceptor were determined with cells treated with only HF488 A $\beta 42$ or only HF647 A $\beta 42$, respectively. The cell autofluorescence was measured in a non-treated control group. The image analysis was processed using the ImageJ plug-in PixFRET.

Primary hippocampal neurons (21 days in vitro) were incubated in the presence of $500 \mathrm{nM} H F 488 \mathrm{~A} \beta 42$ and/or $500 \mathrm{nM}$ HF647 A $\beta 42$ for $24 \mathrm{~h}$ and imaged by a confocal microscope equipped with an Airyscan detector. Both HF488 A $\beta 42$ and HF647 A $\beta 42$ were accumulated in the vesicles of the soma (Figure $2 \mathrm{~b}-\mathrm{c}$ ). After correction, a FRET signal was found in most of the $A \beta$-containing vesicles (Figure $2 \mathrm{~d}-\mathrm{e}$ ).

The corrected FRET can still be affected by the concentration of fluorophores. To get normalized FRET, the corrected FRET value was divided by the square root of the product of donor and acceptor intensities in each pixel. Figure $2 \mathrm{f}$ shows the normalized FRET of the vesicles in the soma of neuron.

Next, to identify the subcellular location where $A \beta$ is accumulated, we used the lysosomal marker SiR-lysosome in neurons that were treated with $1000 \mathrm{nM} \mathrm{A} \beta 42$ for $24 \mathrm{~h}$. Most of $\mathrm{A} \beta$-containing vesicles were colocalized with the lysosomal marker (Figures 3 and S4). This suggests that $\mathrm{A} \beta 42$ was internalized and oligomerized into lysosomes and late endosomes. 

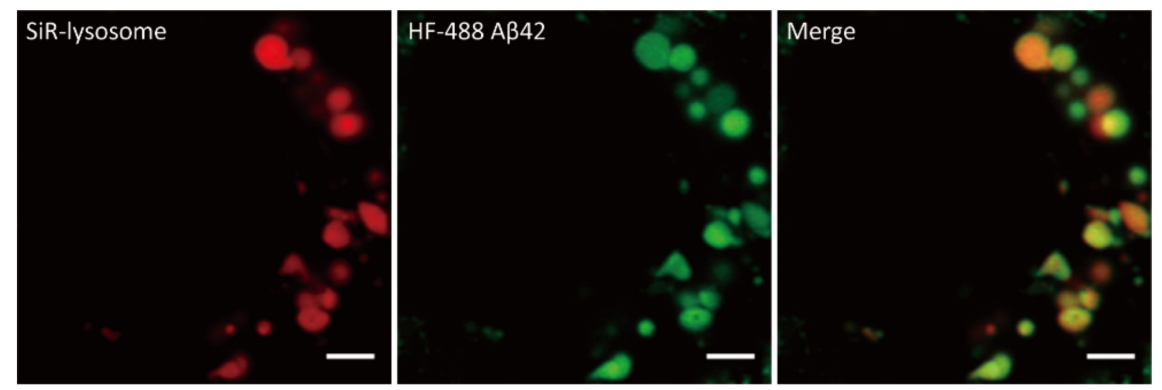

Figure 3. Internalized A $\beta 42$ in primary neurons. DIV 21 neurons were treated with $1000 \mathrm{nM} \mathrm{HF} 488$ A $\beta 42$ (green) for $24 \mathrm{~h}$. SiR-lysosome (red) was used to label lysosome and late endosomes. Scale bar: $2 \mu \mathrm{m}$.

\subsection{A 442 Oligomerization Occurs at Both Surface and Vesicles of Neurons}

A comparison of $A \beta$ oligomerization in vesicles distributed in soma with vesicles in neurites showed no significant differences $(p>0.05$, Figure 4). The weak $A \beta$ signal on the cell surface was difficult to distinguish from the background while imaging the vesicles with high intensities in soma without oversaturation. Thus, higher laser power and detector gain were applied to measure the $A \beta$ aggregation on the cell surface. The normalized FRET value of $A \beta 42$ on the neuron surface or nearabout could be several times higher than in vesicles of soma and neurites (Figure 5). This is consistent with the group treated with lower $A \beta$ concentrations (Figure S5). Similar strong oligomerization was also observed along the neurites, although we did not identify if it came from the surface of neurites.
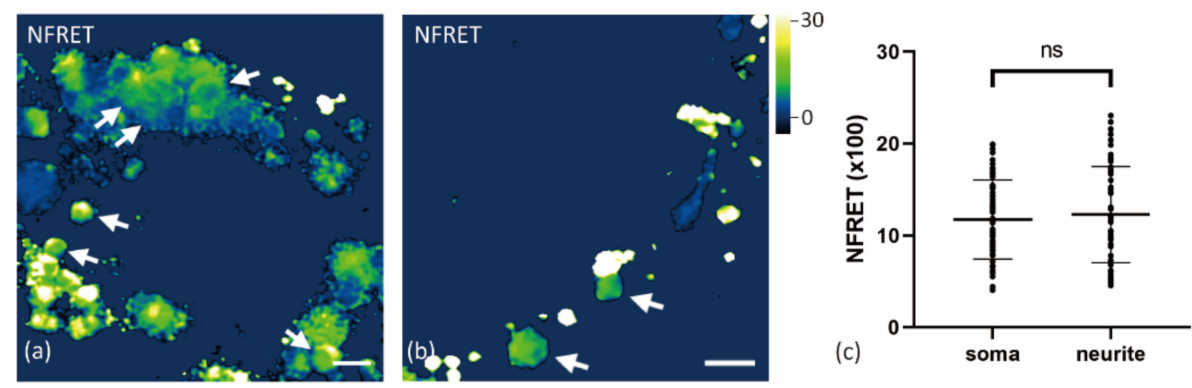

Figure 4. Comparison of FRET between $A \beta$ vesicles in soma and neurites. Primary neurons were treated with $500 \mathrm{nM} \mathrm{HF} 488 \mathrm{~A} \beta 42$ and $500 \mathrm{nM}$ HF647 A $\beta 42$ for 24 h. (a) Normalized FRET (NFRET) image of $A \beta$ vesicles (white arrow) in soma. (b) NFRET image of $A \beta$ vesicles (white arrow) in neurites. (c) NFRET values of 63 vesicles ( 1 cell per image from 8 images) in soma and 64 vesicles (16 images) in neurite were compared using the Mann-Whitney test $(p>0.05)$. Data are mean \pm S.D. Scale bar: $2 \mu \mathrm{m}$.
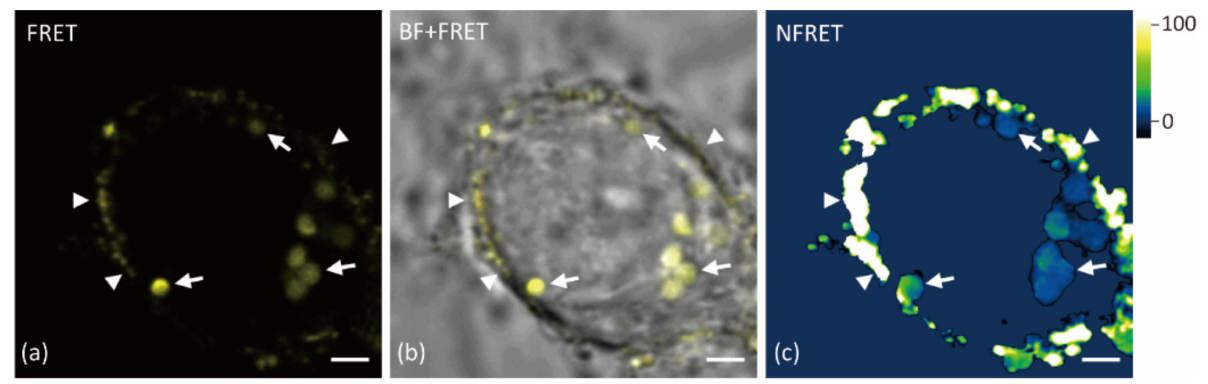

Figure 5. FRET images of $A \beta$ on the cell surface. Primary neurons were treated with 500 nM HF488 A $\beta 42+500$ nM HF647 A $\beta 42$ for 24 h. (a) FRET channel; (b) merged image of brightfield (BF) and FRET channel; (c) normalized FRET image. The white arrow indicates the A $\beta$ vesicles in soma, and the arrowhead indicates the $\mathrm{A} \beta$ on the cell surface. Scale bar: $2 \mu \mathrm{m}$. 


\subsection{Internalized A $\beta 42$ Oligomerization in Soma Is Concentration-Dependent}

To further confirm the association between $\mathrm{A} \beta 42$ oligomerization and concentration in soma, primary neurons were treated with three different $A \beta$ concentrations (HF488 $\mathrm{A} \beta 42$ and HF647 $\mathrm{A} \beta 42$ at 1:1 ratio; 250, 500, and $1000 \mathrm{nM} \mathrm{A} \beta$ in total) for $24 \mathrm{~h}$. As shown in Figure 6, A $\beta$ oligomerization occurred in all the three groups, and increased with extracellular $A \beta 42$ concentration. We also found a negative correlation between the diameter of the $A \beta$ vesicles and the normalized FRET level in two cell groups treated with higher $\mathrm{A} \beta$ concentration. The smaller vesicles tended to present higher oligomerization levels in cells treated with $1000 \mathrm{nM} \mathrm{A} \beta 42(\mathrm{r}=-0.46, p<0.05)$ and $500 \mathrm{nM} \mathrm{A} \beta 42(\mathrm{r}=-0.28$, $p<0.05)$. There was no significant correlation in the $250 \mathrm{nM}$ group $(\mathrm{r}=0.23, p>0.05)$.
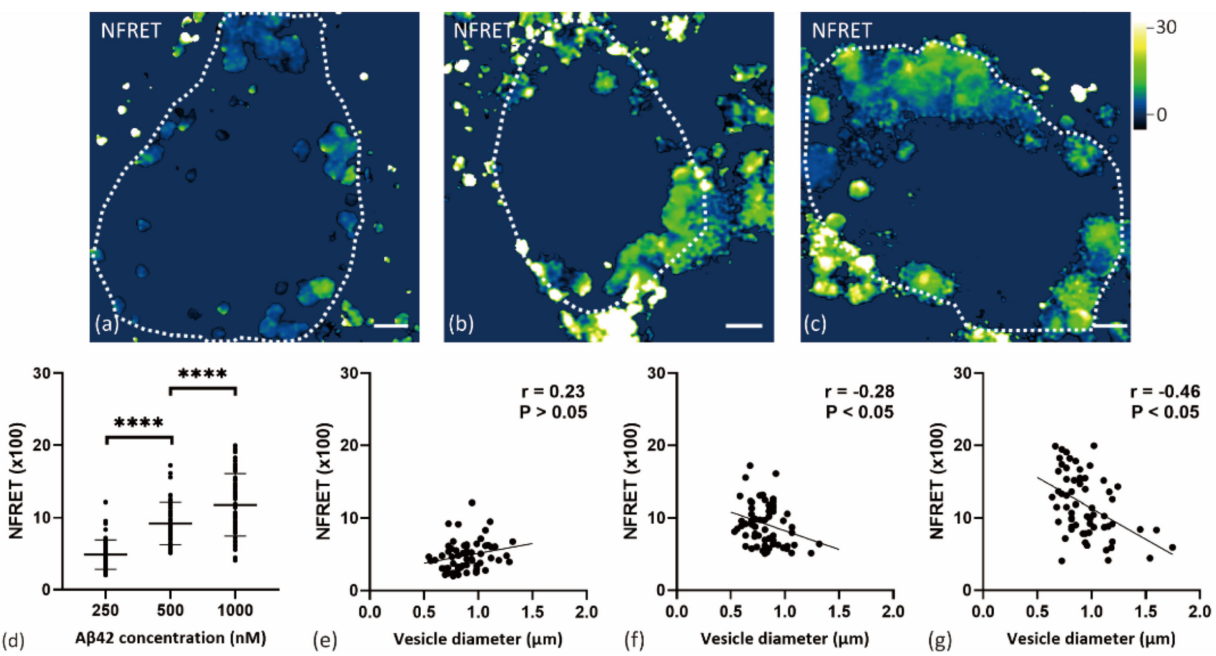

Figure 6. Internalized A $\beta 42$ oligomerization in neuronal soma. (a-c) Normalized FRET (NFRET) image of vesicles in soma. Primary neurons were treated with $250 \mathrm{nM} \mathrm{A} \beta 42$, (a) or $500 \mathrm{nM} \mathrm{A} \beta 42$, (b) or $1000 \mathrm{nM} \mathrm{A} \beta 42$ (c) for $24 \mathrm{~h}$. (d) NFRET values of 63 vesicles (1 cell per image from on the average 8 images) from each group were calculated and compared using the ANOVA test $(p<0.0001)$. Data are mean \pm S.D.; $(\mathbf{e}-\mathbf{g})$ Pearson correlation of $A \beta$ vesicle size and NFRET values in neurons treated with $250 \mathrm{nM} \mathrm{A} \beta 42$, (e) or $500 \mathrm{nM} \mathrm{A} \beta 42$, (f) or $1000 \mathrm{nM} \mathrm{A} \beta 42$ (g) for $24 \mathrm{~h}$. Scale bar: $2 \mu \mathrm{m}$. $* * * *$, indicates $p<0.0001$.

\subsection{Internalized $A \beta 42$ Oligomerizes in Neurons With Higher Efficiency Than A $\beta 40$}

Finally, we treated primary neurons with fluorescently labeled $A \beta 40$, and compared the polymerization of internalized $A \beta 40$ and $A \beta 42$. Confocal image analysis showed that more $A \beta 42$ were taken up into neurons than $A \beta 40$ using the same concentrations for $24 \mathrm{~h}$, and the $\mathrm{A} \beta 42$ oligomerization level was significantly higher as well (Figure S6).

Because the difference of oligomerization could be due to different $A \beta$ concentrations in vesicles, we then compared vesicles with similar intensity of $A \beta 42$ and $A \beta 40$. A $\beta$ concentration in vesicles of cells treated with $1000 \mathrm{nM}$ A $\beta 40$ for $24 \mathrm{~h}$ was comparable to some of those treated with $250 \mathrm{nM} \mathrm{A} \beta 42$ (Figure $7 \mathrm{a}, \mathrm{b}$ ). Therefore, we selected vesicles with similar $A \beta$ concentrations from these two groups and compared their normalized FRET level. $A \beta 42$ treatment was found to induce more oligomerization than $A \beta 40$, under similar $A \beta$ concentrations in vesicles (Figure $7 \mathrm{c}-\mathrm{f}$ ).

Figure 8 shows the scatter plot of normalized FRET values and $A \beta$ concentration in the vesicles of cells treated with different $A \beta$ concentrations. The aggregation of both $A \beta 42$ and $A \beta 40$ showed strong trends to increase with their concentrations in vesicles. Despite lower external concentration, $A \beta 42$ still aggregated to a higher extent than $A \beta 40$. 

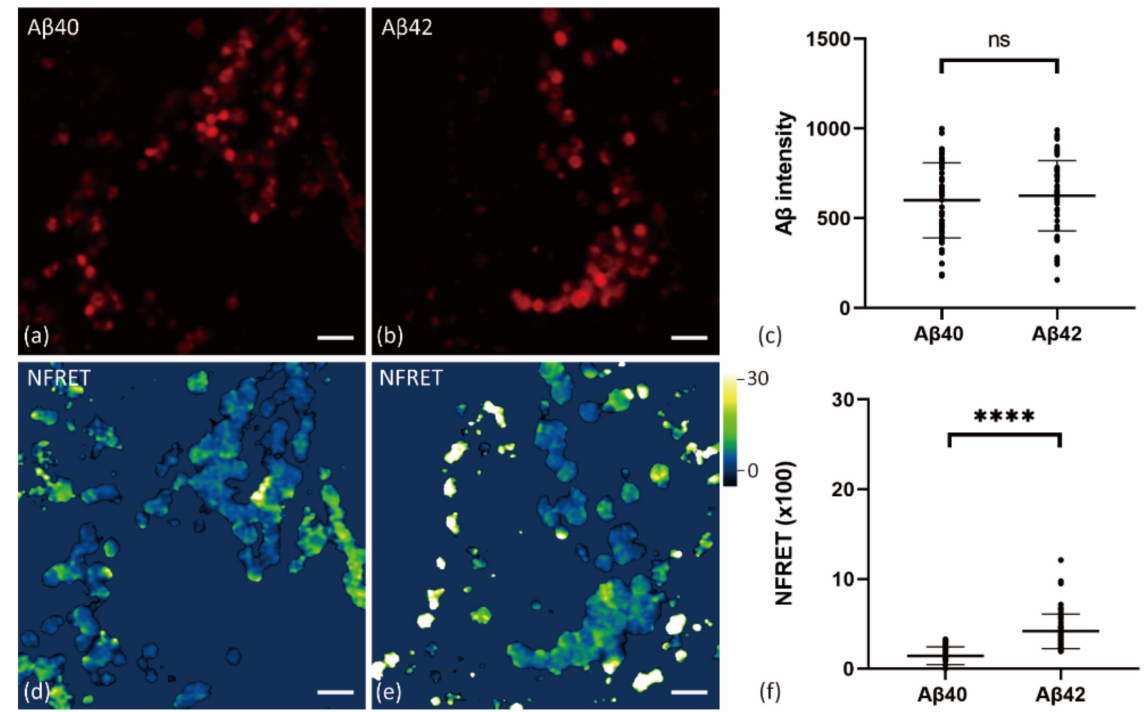

Figure 7. FRET images of neurons treated with $A \beta 40$ or $A \beta 42$. Primary neurons were treated with $1000 \mathrm{nM} \mathrm{A} \beta 40(\mathbf{a}, \mathbf{d})$ or $250 \mathrm{nM} \mathrm{A} \beta 42$ (b,e) for $24 \mathrm{~h}$ (HF488 A $\beta 42$ and HF647 A $\beta 42$ at 1:1 ratio). $(\mathbf{a}-\mathbf{b})$ Confocal images of $\mathrm{A} \beta$ vesicles (HF647) in primary neurons; the same acquisition and display settings were used for both groups. (c) A $\beta$ intensity (HF647) of selected vesicles in neurons treated with $1000 \mathrm{nM} \mathrm{A} \beta 40(n=54,1$ cell per image from 8 images) and $250 \mathrm{nM} \mathrm{A} \beta 42(n=65,1$ cell per image from 9 images) were compared using the Mann-Whitney test $(p>0.05)$. (d-e) Normalized FRET (NFRET) images of $A \beta$ vesicles in primary neurons. (f) NFRET values of selected $A \beta$ vesicles in neuron treated with $\mathrm{A} \beta 40$ and $\mathrm{A} \beta 42$ were compared using the Mann-Whitney test $(p<0.0001)$. Data are mean \pm S.D. Scale bar: $2 \mu \mathrm{m}$. ${ }^{* * *}$, indicates $p<0.0001$. "ns" indicates $p>0.05$.

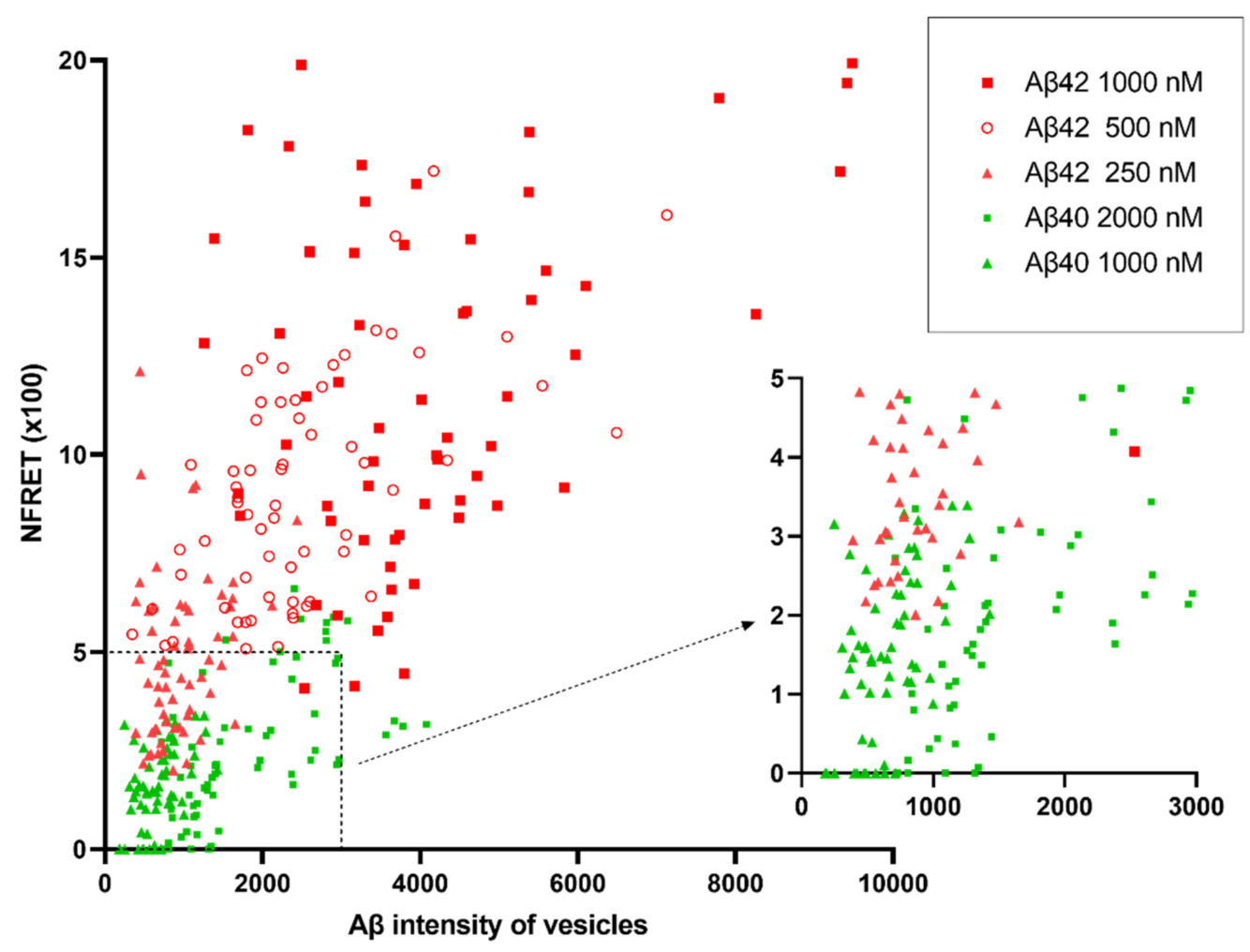

Figure 8. Scatter plot of normalized FRET (NFRET) and A $\beta$ intensity (HF647) in vesicles of neuronal soma. Primary neurons were treated with different concentrations of $A \beta 40$ (green) or $A \beta 42$ (red) for $24 \mathrm{~h}$ (HF488 A $\beta$ and HF647 A $\beta$ at 1:1 ratio). The right graph shows enlarged details in the dashed box. 


\section{Discussion}

$\mathrm{A} \beta$ aggregation is thought to have a crucial role in the progression of $\mathrm{AD}$. However, the detection of $A \beta$ oligomers is challenging due to it lacking stability and being easily influenced during analysis [18]. FRET has been used to measure molecular distances at the nanoscale for a long time. Here, by measuring FRET with a confocal microscope with an Airyscan unit, we confirmed that extracellular monomeric $A \beta$ could be internalized into the murine hippocampal neurons and oligomerized in lysosomes and/or late endosomes. The high normalized FRET signal on the cell surfaces indicated part of the A $\beta$ oligomers might have already formed before getting into lysosomes/late endosomes.

It has been suggested that intraneuronal $A \beta$ accumulation could be an early event in the pathogenesis of $\mathrm{AD}$ [4]. In this study, extracellular $\mathrm{A} \beta$ is taken up by neurons and oligomerized intracellularly, making it a possible source of intraneuronal $A \beta$ aggregation. We observed strong $A \beta$ oligomerization occurred in primary neurons treated with only $250 \mathrm{nM}$ A $\beta 42$ for $24 \mathrm{~h}$. Previously, Hu et al. did not detect A $\beta$ aggregation in SHSY5Y cells treated with $500 \mathrm{nM} \mathrm{A} \beta 42$ or below for five days, using agarose gel electrophoresis-SDS [16]. This might be due to the cellular uptake difference of $A \beta$, or because $A \beta$ oligomers are SDS-instable species and this analysis could result in disassembly of oligomers [13].

In our study, $A \beta$ in neurons aggregated in a concentration-dependent manner. This is consistent with previous studies in SHSY5Y cells and in buffers [16,19]. Besides, the size of $A \beta$ vesicles also showed a significant negative correlation with aggregation in cells with $24 \mathrm{~h}$ treatment of higher $A \beta$ concentration, but not in lower concentration. We suggest that cells with lower $A \beta$ concentration treatment could be at an earlier stage of uptake at $24 \mathrm{~h}$, compared with those with high concentrations.

The $A \beta$ oligomer distribution in neurons is also interesting. It is reported that more lysosomes are located in soma than in neurites [20]. However, the oligomerization level in lysosomes/late endosomes of soma and neurites seems similar. We also identified the strongest normalized FRET signal on the neuron surface based on the following consideration. First, the fluorescence on the cell surface lacked movement during imaging, different from those in the cellular organelles. Secondly, such distinctly high normalized FRET was observed only on the cell border and along neurites, but not in soma. Besides, it has been reported that $A \beta$ forms oligomers on lipid rafts in the cell membrane [21].

Regarding intraneuronal $A \beta 42$ aggregation, another question is where this progress begins. First, we detected only little A $\beta 42$ oligomerization at a $1000 \mathrm{nM}$ concentration in the cell culture medium. Secondly, we noticed high $A \beta$ oligomerization on the surface of neurons. The values of normalized FRET were several times higher than in the soma. The normalized FRET could be affected by the distance-dependent FRET efficiency and the ratio of "FRET" donor-acceptors to total donors and acceptors [22]. In this study, such a large difference in the normalized FRET between soma and membrane cannot be explained by the FRET efficiency. Thus, we infer that lysosomes/late endosomes in soma contained non-FRET monomeric $A \beta 42$. The concentration of $A \beta 42$ in those vesicles is much higher than in extracellular space [16]. Low $\mathrm{pH}$ in the lysosomes/late endosomes might promote $\mathrm{A} \beta$ aggregation $[19,23]$. Considering the high concentration and low $\mathrm{pH}, \mathrm{A} \beta$ monomers in these vesicles are more likely to be taken up extracellularly rather than depolymerized from oligomers. We suggest that at least part of $A \beta$ is internalized in the monomeric form, then aggregates in the late endosomes/lysosomes. However, it is possible that part of $A \beta$ oligomerizes at the cell surface before entering late endosomes/lysosomes. It is also possible that oligomers that formed in neurons are released and then reinternalized to the endo-lysosomal system.

Compared with $A \beta 42, A \beta 40$ is less prone to form fibrils [24]. In the present study, $A \beta 42$ aggregation dominance in neurons was partly owing to the cellular uptake preference, which resulted in high $A \beta 42$ concentration in vesicles. Under similar concentrations in vesicles, $A \beta 42$ still oligomerized more than $A \beta 40$, however the difference is less than we expected. Although normally $A \beta 42$ is present at lower concentrations than $A \beta 40$ [25], it can induce more oligomers due to the enhanced cellular uptake and its strong aggregation 
tendency. This novel finding is in line with our previous finding showing that elevated levels of $\mathrm{A} \beta 42$ in neurons is a risk in brains of those with AD [9]. Thus, we hypothesize that $A \beta 42$ accumulation in neurons by enhanced uptake is an early pathogenic event in $\mathrm{AD}$, and that inhibition of this process could be a pharmacological strategy for treating $\mathrm{AD}$.

In this study, we chose sensitized emission FRET for several reasons. It is simple, nondestructive, suitable for live imaging, and our study also fitted the stoichiometry of 1:1 donor and acceptor. The fast and sensitive Airyscan unit allowed us to detect FRET signals in moving vesicles, but it also detected unwanted autofluorescence. To minimize the caused false positive FRET, autofluorescence was measured from the non-treated vesicles that present the fluorescence. However, detected autofluorescence existed only in part of the vesicles, so background subtraction from the whole image might underestimate the FRET of those compartments with less autofluorescence, or even result in false negative values. In addition, although the bleed-through parameters were determined carefully, it was still difficult to measure accurate FRET data quantitatively with sensitized emission FRET, especially when the FRET signal was weak. This limits the use of sensitized emission FRET in some applications, such as determining the lowest $\mathrm{A} \beta$ concentration or the earliest timepoint to aggregate. Nevertheless, live cell FRET imaging can provide in situ detection with almost no intervention, making it a robust and unique technique for oligomerization measurement, when designed properly and conducted under controlled conditions.

In summary, our study suggests that the $A \beta 42$ monomer can be internalized in neurons and oligomerized in the late endosome/lysosomes with high efficiency. In such a way, A $\beta 42$ oligomers might accumulate gradually in neurons over decades of a lifetime, and cause the neuronal dysfunction.

\section{Materials and Methods}

\subsection{Synthetic Human $A \beta$ Peptide and Preparation}

$\mathrm{A} \beta$ (1-40) and A $\beta$ (1-42) peptides, labeled with HiLyte ${ }^{\mathrm{TM}}$ Fluor 488 or HiLyte ${ }^{\mathrm{TM}}$ Fluor 647 at the N-termini, were purchased from AnaSpec (BioNordika, Solna, Sweden). A $\beta$ peptides were reconstituted by adding $40-50 \mu \mathrm{l} 1 \% \mathrm{NH}_{4} \mathrm{OH}$ to $0.1 \mathrm{mg}$ dry peptide and diluted to $1 \mathrm{mg} / \mathrm{ml}$ with $\mathrm{PBS}$, then aliquoted and stored at $-20^{\circ} \mathrm{C}$.

\subsection{Primary Neuron Culture}

Hippocampal neurons were isolated from the hippocampi of E16.5 C57/BL6 mice embryo brains, seeded on the inner $10 \mathrm{~mm}$ microwell of poly-d-lysine-coated glass bottoms of P35G-1.5-10-C culture dishes (MatTek Corporation), as described previously [26]. Cortical neurons were seeded at the pre-coated edges of the plate as a support layer. Both hippocampal and cortical neurons were grown in selective Neurobasal medium containing $2 \%$ B27 (Invitrogen) and 1\% L-glutamine (Invitrogen) at $37^{\circ} \mathrm{C}$ in a cell incubator (humidified, $5 \% \mathrm{CO}_{2}$ ), and cultured for 21 days in vitro (DIV).

\subsection{Lysosomes/Late Endosomes Labeling in Live Cell Imaging}

Lysosomes/late endosomes were labeled using SiR-Lysosome (Spirochrome AG). Primary neurons were treated with $1000 \mathrm{nM} \mathrm{SiR}$-Lysosome at $37^{\circ} \mathrm{C}, 5 \% \mathrm{CO}, 0.5 \mathrm{~h}$ before imaging.

\subsection{Confocal Microscope and FRET Measurements}

Confocal and FRET images were acquired using a Zeiss LSM 800 confocal microscope with Airyscan detector, using a Plan-Apochromat $63 \times / 1.4$ oil-immersion objective and the 488 and $640 \mathrm{~nm}$ laser lines as excitation source, unless specified otherwise. For FRET measurements, the laser power and detector gain for all channels were fixed through the whole experiment. Each channel was subsequently scanned by a 32-channel gallium arsenide phosphide photomultiplier tube (GaAsP-PMT) Airyscan detector. The acquired FRET images were $452 \times 452$ pixels (scan zoom: $5.0 \times$ ) with a scan speed of $2.2 \mu \mathrm{s} /$ pixel 
(pixel size: $0.043 \mu \mathrm{m}$ ). The pinhole for the fluorescence channel was $5.0 \mathrm{AU}$; for the brightfield channel it was 1.0 AU. Five to ten images were captured per cell culture dish.

The cells were incubated with either HF488 A $\beta$ or HF647 A $\beta$, or an equimolar mixture of them. The donor control group was imaged sequentially in donor emission (488 nm excitation, 490-580 nm emission) and donor bleed-through (488 nm excitation, 650-700 nm emission). The acceptor control group included acceptor emission (640 $\mathrm{nm}$ excitation, 650-700 $\mathrm{nm}$ emission) and acceptor crosstalk (488 $\mathrm{nm}$ excitation, 650-700 nm emission). The FRET group contained three image sets: donor emission, acceptor emission, and FRET channel (488 $\mathrm{nm}$ excitation, $650-700 \mathrm{~nm}$ emission). The non-treated neurons group that was used to detect the cell autofluorescence and background shared the same settings as the FRET group.

FRET analysis was performed using the ImageJ plug-in PixFRET as previously described [27]. Concisely, spectral bleed-through was first determined in each image acquired from the donor control group and acceptor control group, by using a constant model in PixFRET. A Gaussian blur value of 2.0 was applied during the determination. By applying PixFRET computation, the original FRET images were then corrected from the spectral bleed-through and generated the FRET and normalized FRET image. "FRET/sqrt (Donor*Acceptor)" was selected as the output of normalized FRET, in which values for each pixel were divided by the square root of donor and acceptor intensities from same pixels. Gaussian blur (2.0) and threshold (1.0) values were set for all the images. To clearly distinguish the low and high values, normalized FRET images were displayed in a lookup table "Green Fire Blue". For comparison, the negative normalized FRET values were set to 0 . The quantification of normalized FRET values and $A \beta$ intensity in vesicles were measured with ImageJ. $A \beta$ intensity was calculated with HF647 A $\beta$ because acceptor emission is not affected by FRET. The sizes of $A \beta$ vesicles were determined in the fluorescence channel; the vesicles were selected using the oval tool and the diameter was calculated from the measured area of vesicles by ImageJ ("Analyze-Measure").

FCS, FCCS and FCS-FRET measurements were recorded using a Zeiss 780 confocal laser scanning microscope equipped for FCS, with a Zeiss water immersion objective, C-Apochromat $40 \times / 1.2 \mathrm{NA}$. The measurements have been described previously [17]. In this study, FRET-FCS was performed on the cell-free neuronal culture medium with $24 \mathrm{~h}$ treatment of $500 \mathrm{nM} \mathrm{HF} 488 \mathrm{~A} \beta 42$ and $500 \mathrm{nM}$ HF647 A $\beta 42$. The diffusion time $(\tau)$ refers to the mean time of fluorescent particles passing through the detection focus.

\subsection{Statistics}

Statistical tests were performed using the GraphPad Prism 8 software. Normality was assessed using D'Agostino and Pearson tests. The non-parametric Mann-Whitney test was used for the data that were not normally distributed. One-way ANOVA was used for comparison of three groups. Correlation analysis was tested by the Pearson method. When the $p$ value was less than 0.0001 , it is stated as "****", and "**" for $p<0.05$. If the $\mathrm{p}$ was greater than 0.05 , it is stated as $p>0.05$ or "ns". The data were obtained from two primary culture preparations. For statistical analysis, 408 vesicles from at least 56 images/cells taken from ten culture dishes derived from two primary neuron preparations were analyzed.

Supplementary Materials: The following are available online at https:/ / www.mdpi.com/article/10 $.3390 /$ ijms22094530/s1.

Author Contributions: Conceptualization, Y.G., S.S.-W. and L.O.T.; methodology, Y.G., S.W., S.S.W. and L.O.T.; formal analysis, Y.G. and S.W.; writing-original draft preparation, Y.G., and S.W.; writing-review and editing, Y.G., S.W., S.S.-W. and L.O.T.; visualization, Y.G. and S.W.; supervision, B.W., S.S.-W. and L.O.T.; funding acquisition, S.S.-W., B.W. and L.O.T. All authors have read and agreed to the published version of the manuscript.

Funding: This work was supported by grants from Margaretha af Ugglas' foundation (B.W., S.S.-W.), Swedish Alzheimer foundation (L.T. and S.S.-W.), Swedish Research Council (L.O.T.), China Scholarship Council, Stiftelsen för Gamla Tjänarinnor, and the Gun and Bertil Stohnes Foundation. 
Institutional Review Board Statement: This study was approved by Linköping ethical committee (ID 1241, date of approval 25 January 2018).

Data Availability Statement: The data presented in this study are available on request from the corresponding author.

Acknowledgments: We acknowledge help from Sylvie Le Guyader and Gabriela Imreh from the Live Cell Imaging facility, Karolinska Institutet, Sweden, supported by grants from the Knut and Alice Wallenberg Foundation, the Swedish Research Council, the Centre for Innovative Medicine and the Jonasson Centre at the Royal Institute of Technology, Sweden.

Conflicts of Interest: The authors declare no conflict of interest.

\section{References}

1. Ballard, C.; Gauthier, S.; Corbett, A.; Brayne, C.; Aarsland, D.; Jones, E. Alzheimer's Disease. Lancet 2011, 377, 1019-1031. [CrossRef]

2. 2020 Alzheimer's Disease Facts and Figures. Alzheimers Dement. 2020, 16, 391-460. [CrossRef] [PubMed]

3. Viola, K.L.; Klein, W.L. Amyloid $\beta$ Oligomers in Alzheimer's Disease Pathogenesis, Treatment, and Diagnosis. Acta Neuropathol. 2015, 129, 183-206. [CrossRef]

4. LaFerla, F.M.; Green, K.N.; Oddo, S. Intracellular Amyloid- $\beta$ in Alzheimer's Disease. Nat. Rev. Neurosci. 2007, 8, 499-509. [CrossRef]

5. Turner, R.S.; Suzuki, N.; Chyung, A.S.; Younkin, S.G.; Lee, V.M.-Y. Amyloids Beta40 and Beta42 Are Generated Intracellularly in Cultured Human Neurons and Their Secretion Increases with Maturation. J. Biol. Chem. 1996, 271, 8966-8970. [CrossRef] [PubMed]

6. Tienari, P.J.; Ida, N.; Ikonen, E.; Simons, M.; Weidemann, A.; Multhaup, G.; Masters, C.L.; Dotti, C.G.; Beyreuther, K. Intracellular and Secreted Alzheimer Beta-Amyloid Species Are Generated by Distinct Mechanisms in Cultured Hippocampal Neurons. Proc. Natl. Acad. Sci. USA 1997, 94, 4125-4130. [CrossRef]

7. Walsh, D.M.; Selkoe, D.J. A $\beta$ Oligomers-A Decade of Discovery. J. Neurochem. 2007, 101, 1172-1184. [CrossRef] [PubMed]

8. Karisetty, B.C.; Bhatnagar, A.; Armour, E.M.; Beaver, M.; Zhang, H.; Elefant, F. Amyloid- $\beta$ Peptide Impact on Synaptic Function and Neuroepigenetic Gene Control Reveal New Therapeutic Strategies for Alzheimer's Disease. Front. Mol. Neurosci. 2020, 13. [CrossRef]

9. Hashimoto, M.; Bogdanovic, N.; Volkmann, I.; Aoki, M.; Winblad, B.; Tjernberg, L.O. Analysis of Microdissected Human Neurons by a Sensitive ELISA Reveals a Correlation between Elevated Intracellular Concentrations of Abeta42 and Alzheimer's Disease Neuropathology. Acta Neuropathol. 2010, 119, 543-554. [CrossRef]

10. Aoki, M.; Volkmann, I.; Tjernberg, L.O.; Winblad, B.; Bogdanovic, N. Amyloid $\beta$-Peptide Levels in Laser Capture Microdissected Cornu Ammonis 1 Pyramidal Neurons of Alzheimer's Brain. NeuroReport 2008, 19, 1085-1089. [CrossRef]

11. Nag, S.; Sarkar, B.; Bandyopadhyay, A.; Sahoo, B.; Sreenivasan, V.K.A.; Kombrabail, M.; Muralidharan, C.; Maiti, S. Nature of the Amyloid- $\beta$ Monomer and the Monomer-Oligomer Equilibrium. J. Biol. Chem. 2011, 286, 13827-13833. [CrossRef]

12. Hayden, E.Y.; Teplow, D.B. Amyloid $\beta$-Protein Oligomers and Alzheimer's Disease. Alzheimers Res. Ther. 2013, 5, 60. [CrossRef]

13. Bruggink, K.A.; Müller, M.; Kuiperij, H.B.; Verbeek, M.M. Methods for Analysis of Amyloid- $\beta$ Aggregates. J. Alzheimers Dis. 2012, 28, 735-758. [CrossRef]

14. Algar, W.R.; Hildebrandt, N.; Vogel, S.S.; Medintz, I.L. FRET as a Biomolecular Research Tool—Understanding Its Potential While Avoiding Pitfalls. Nat. Methods 2019, 16, 815-829. [CrossRef]

15. Wesén, E.; Gallud, A.; Paul, A.; Lindberg, D.J.; Malmberg, P.; Esbjörner, E.K. Cell Surface Proteoglycan-Mediated Uptake and Accumulation of the Alzheimer's Disease Peptide A $\beta(1-42)$. Biochim. Biophys. Acta BBA Biomembr. 2018, 1860, $2204-2214$. [CrossRef] [PubMed]

16. Hu, X.; Crick, S.L.; Bu, G.; Frieden, C.; Pappu, R.V.; Lee, J.-M. Amyloid Seeds Formed by Cellular Uptake, Concentration, and Aggregation of the Amyloid-Beta Peptide. Proc. Natl. Acad. Sci. USA 2009, 106, 20324-20329. [CrossRef]

17. Wennmalm, S.; Chmyrov, V.; Widengren, J.; Tjernberg, L. Highly Sensitive FRET-FCS Detects Amyloid $\beta$-Peptide Oligomers in Solution at Physiological Concentrations. Anal. Chem. 2015, 87, 11700-11705. [CrossRef] [PubMed]

18. Mroczko, B.; Groblewska, M.; Litman-Zawadzka, A.; Kornhuber, J.; Lewczuk, P. Amyloid $\beta$ Oligomers (A $\beta O s)$ in Alzheimer's Disease. J. Neural Transm. 2018, 125, 177-191. [CrossRef] [PubMed]

19. Burdick, D.; Soreghan, B.; Kwon, M.; Kosmoski, J.; Knauer, M.; Henschen, A.; Yates, J.; Cotman, C.; Glabe, C. Assembly and Aggregation Properties of Synthetic Alzheimer's A4/Beta Amyloid Peptide Analogs. J. Biol. Chem. 1992, 267, 546-554. [CrossRef]

20. Kulkarni, V.V.; Maday, S. Neuronal Endosomes to Lysosomes: A Journey to the Soma. J. Cell Biol. 2018, 217, 2977-2979. [CrossRef]

21. Kim, S.-I.; Yi, J.-S.; Ko, Y.-G. Amyloid $\beta$ Oligomerization Is Induced by Brain Lipid Rafts. J. Cell. Biochem. 2006, 99, 878-889. [CrossRef]

22. Xia, Z.; Liu, Y. Reliable and Global Measurement of Fluorescence Resonance Energy Transfer Using Fluorescence Microscopes. Biophys. J. 2001, 81, 2395-2402. [CrossRef] 
23. Su, Y.; Chang, P.-T. Acidic PH Promotes the Formation of Toxic Fibrils from $\beta$-Amyloid Peptide. Brain Res. 2001, 893, $287-291$. [CrossRef]

24. Jarrett, J.T.; Berger, E.P.; Lansbury, P.T. The Carboxy Terminus of the.Beta. Amyloid Protein Is Critical for the Seeding of Amyloid Formation: Implications for the Pathogenesis of Alzheimer's Disease. Biochemistry 1993, 32, 4693-4697. [CrossRef]

25. Asami-Odaka, A.; Ishibashi, Y.; Kikuchi, T.; Kitada, C.; Suzuki, N. Long Amyloid Beta-Protein Secreted from Wild-Type Human Neuroblastoma IMR-32 Cells. Biochemistry 1995, 34, 10272-10278. [CrossRef] [PubMed]

26. Yu, Y.; Jans, D.C.; Winblad, B.; Tjernberg, L.O.; Schedin-Weiss, S. Neuronal A $\beta 42$ Is Enriched in Small Vesicles at the Presynaptic Side of Synapses. Life Sci. Alliance 2018, 1, e201800028. [CrossRef] [PubMed]

27. Feige, J.N.; Sage, D.; Wahli, W.; Desvergne, B.; Gelman, L. PixFRET, an ImageJ Plug-in for FRET Calculation That Can Accommodate Variations in Spectral Bleed-Throughs. Microsc. Res. Tech. 2005, 68, 51-58. [CrossRef] 